\title{
THE RELATIVE CONTRIBUTIONS OF MYOCARDIAL WALL THICKNESS AND ISCHEMIA TO ULTRASONIC MYOCARDIAL INTEGRATED BACKSCATTER DURING EXPERIMENTAL ISCHEMIA
}

\author{
H. Rijsterborgh, F. Mastik, C. T. Lancée, L. M. A. Sassen, \\ P. D. VERDOUW, J. ROELANDT and N. BOM \\ Thoraxcentre, Erasmus University Rotterdam and Interuniversity Cardiology Institute of The Netherlands
}

\author{
(Received 12 February 1990; in final form 14 May 1990)
}

\begin{abstract}
The purpose of this study was to assess the empirical relationship between myocardial integrated backscatter (IB) and myocardial wall thickness (WT) in normal myocardium. A second object was to estimate the additional contribution to acute ischemic integrated backscatter levels given this relationship. Myocardial IB measurements and simultaneous myocardial WT measurements were made in 16 open-chested pigs with intact coronary circulation (normal myocardium) and $10 \mathrm{~min}$ after the flow in the left anterior descending coronary artery had been reduced to $20 \%$ of its baseline value (ischemic myocardium). Measurements were made 50 times during one cardiac cycle and averaged over 10 cardiac cycles. IB and WT measurements were normalized with respect to the nonischemic end-diastolic values. The relationship between $I B$ and $W T$ in normal myocardium was estimated in every individual pig by simple linear regression. Estimates of IB during ischemia were calculated on the basis of this relationship and the ischemic WT measurements. Differences of the estimator and the actual measurement made during ischemia depict the actual contribution of the state of acute ischemia, without the influence of WT. The slope of the relationship between IB and WT during normal myocardial contraction ranged from -0.16 to $0.03 \mathrm{~dB} / \%$ (mean $=-0.036 \mathrm{~dB} / \%, \mathrm{SD}=0.06 \mathrm{~dB} / \%$ ). The additional contribution of ischemia ranged from -3.84 to $5.56 \mathrm{~dB}$ (mean $=0.31 \mathrm{~dB}, \mathrm{SD}=2.72 \mathrm{~dB}$ ). It was concluded that the average contribution of ischemia to IB measurements is insignificant if the IB dependency on WT is removed from the data and that the higher level of ischemic IB measurements can be explained by the decrease in wall thickness during ischemia and not by the ischemia itself.
\end{abstract}

Key Words: Ultrasound, Myocardium, Integrated backscatter, Wall thickness, Acute ischemia.

\section{INTRODUCTION}

A well-known feature of ultrasonic myocardial integrated backscatter is its cyclic variation during the cardiac cycle: in normal myocardium end-diastolic integrated backscatter is higher as compared to endsystolic backscatter levels (Miller et al. 1983; Mottley et al. 1984; Wickline et al. 1985). The cyclic variation suggests an inverse relationship between integrated backscatter measurements and myocardial wall thickness. This relationship has been investigated in normal and ischemic myocardial tissue (Wickline et al. 1986; Rijsterborgh et al. 1990) in animal experiments.

Address correspondence to: $H$. Rijsterborgh, Thoraxcenter Ee 2302, Erasmus University Rotterdam, P.O. Box 1738, 3000 DR Rotterdam, The Netherlands.

These investigations are supported by the Netherlands Technology Foundation (STW).
It has been shown previously that integrated backscatter levels in acute ischemic myocardial tissue are higher as compared to integrated backscatter levels measured in normal myocardium (O'Donnell et al. 1979; Miller et al. 1983; Glueck et al. 1985; Rijsterborgh et al. 1990).

After the occlusion of a coronary artery, the two factors which might influence the magnitude of integrated backscatter measurements change simultaneously: the myocardial tissue becomes acutely ischemic and the myocardial wall thickness decreases. The intriguing question therefore remains whether the observed increased levels of integrated backscatter during acute ischemia is related to the decreased wall thickness or the state of ischemia itself.

It was the purpose of this study to assess the magnitude of the relationship between integrated backscatter and myocardial wall thickness and to investigate the relative contributions of wall thickness 
and the presence of acute ischemia to integrated backscatter levels in animal experiments. The relationship between ultrasonic integrated backscatter and myocardial wall thickness was assessed during normal myocardial performance. On the basis of this relationship and the wall thickness measurements taken during acute ischemia, the level of integrated backscatter was estimated and compared with the actual measurements. A significant difference between the estimated backscatter and the actual measurements designates the contribution of the state of acute ischemia.

A computer-based measurement system was applied (Lancée et al. 1988; Rijsterborgh et al. 1990) to obtain simultaneous measurements of myocardial integrated backscatter, myocardial wall thickness and left ventricular pressure.

\section{METHODS}

\section{General}

Animal experiments were performed on young Yorkshire pigs according to the guiding principles for the care and use of animals (Office of Science and Health Reports 1980). The animals ( $n=16,24-30$ $\mathrm{kg}$ ) were sedated with $120 \mathrm{mg}$ azaperone (i.m.) and subsequently anaesthetized with $150 \mathrm{mg}$ of metomidate (i.v.). After intubation, the animals were respirated with a mixture of $30 \%$ oxygen and $70 \%$ nitrous oxide. Anaesthesia was maintained with $160 \mathrm{mg} / \mathrm{kg} / \mathrm{h}$ $\alpha$-chloralose (Merck, Darmstadt, FRG) followed by an infusion of $5 \mathrm{mg} / \mathrm{kg}$ pentobarbitone sodium (Sanofi, Paris, France) via a catheter placed in the superior vena cava. Respiratory rate and tidal volume were adjusted to maintain physiological arterial blood gas values: (mean \pm sem), $7.39 \pm 0.01(\mathrm{pH}), 45$ $\pm 3 \mathrm{mmHg}\left(\mathrm{pCO}_{2}\right), 154 \pm 4 \mathrm{mmHg}\left(\mathrm{pO}_{2}\right)$ and 93 $\pm 2 \%\left(\mathrm{O}_{2}\right.$ saturation), measured with an $\mathrm{ABL}-3$ (Radiometer, Copenhagen, Denmark).

Left ventricular and central aortic pressure were monitored with microtipped catheters (HoneywellPhilips, Best, The Netherlands). After the heart was exposed using a midsternal split, a hydraulic occluder (RE Jones, Silver Spring, MD, USA) was placed around the proximal left anterior descending coronary artery (LADCA) just distal to its first diagonal branch and connected to a $1 \mathrm{~mL}$ syringe (Hamilton Bonaduz, Bonaduz, Switzerland) which was driven by a micrometer. Regional myocardial function was estimated from myocardial wall thickness recordings obtained with the aid of a $4.6 \mathrm{MHz}$ ultrasonic transducer (Krautkramer-Branson, Lewistown, PA, USA) sutured onto the epicardial surface in the middle of the region perfused by the distal part of the LADCA that was to be occluded. The transducer remained at this site during the entire experiment. The transducer was connected to a commercially available echocardiographic ultrasound system (Organon Teknika, Oss, The Netherlands).

\section{Experimental protocol}

After systemic hemodynamics had been stable for at least $30 \mathrm{~min}$ following completion of the surgical procedures, baseline values of systemic hemodynamics and regional myocardial function were obtained. LADCA flow was then gradually reduced by slowly inflating the balloon until regional wall thickening was abolished. Wall thickening remained absent during the episode of ischemia. If necessary, minor adjustments in the degree of flow reduction were performed during the first $5 \mathrm{~min}$ of ischemia, but, thereafter, no further manipulations were allowed (Sassen et al. 1988).

\section{Data acquisition}

The measurements were made at two episodes during the experiments: the baseline measurements were obtained just before the occlusion was applied and after $10 \mathrm{~min}$ of subsequent acute ischemia. The ultrasound transducer (Krautkramer-Branson, Lewistown, PA, USA) was connected to an in-house developed transmitter/wide band amplifier (input impedance $100 \mathrm{Ohm}$, fixed gain $29 \mathrm{~dB},-6 \mathrm{~dB}$ cut-off frequencies 1.2 and $10 \mathrm{MHz}$ ). Amplified ultrasound signals were filtered by a 5 th order $10 \mathrm{MHz}$ low-pass Chebyshev filter and connected to one channel of a dual channel digital oscilloscope (LeCroy 9400), digitizing the time interval of interest $(4.5 \mu \mathrm{s}$ to $24.5 \mu \mathrm{s}$ following the transmitter pulse) with an 8 bit resolution at a sample frequency of $25 \mathrm{MHz}$. Simultaneously, a left ventricular pressure signal was digitized by the oscilloscope. The oscilloscope was interfaced with an IBM computer system (AT-3).

Measurement sequences were controlled by the computer system. At the onset of the pressure curve (begin systole), 62 broadband transmitting pulses (duration approximately $1 \mu \mathrm{s}$; see Fig. 1a) were generated with a repetition rate of 50 times the pig's heart rate. Following the last transmitter pulse, the accumulated high-frequency ultrasound data were stored on disc. Data acquisition of 10 cardiac cycles could be accomplished within $30-40 \mathrm{~s}$. This procedure was repeated for every episode of the study.

\section{Data processing}

Data were processed off-line. Ultrasound power spectra were calculated by a Fourier transformation using an array processor (Data Translation 7020). A 


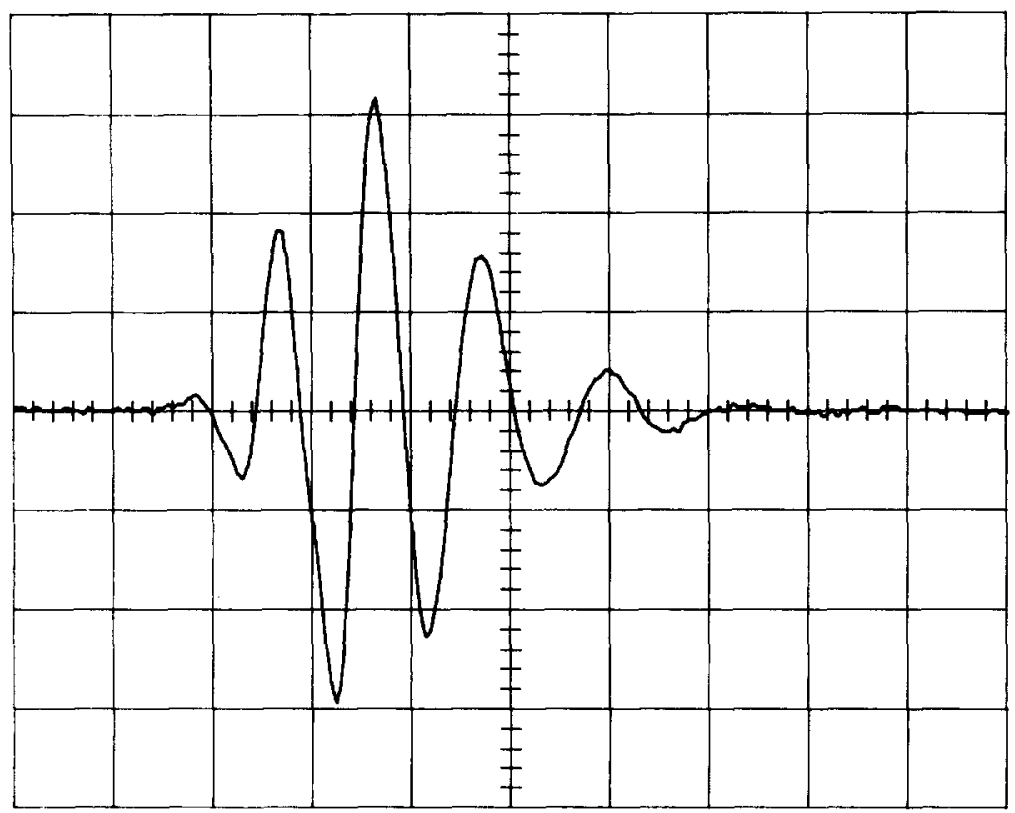

(a)

Fig. 1a. The time domain response of the ultrasound transmitter/receiver system measured at a flat stainless steel reflector at a distance of $5.4 \mathrm{~mm}(7 \mu \mathrm{s})$. The horizontal scale is $0.2 \mu \mathrm{s}$ per division.

split cosine bell window ( $p=0.1$; Bloomfield 1976) of the time interval from 5 to $8 \mu$ s following the transmitter pulse was chosen to select the region of interest and to exclude the reflections of the endocardial wall from the spectra. Spectra were corrected for the frequency response of the transducer and the wide band amplifier by a reference spectrum measured from a flat stainless steel reflector (Fig. 1b). This was

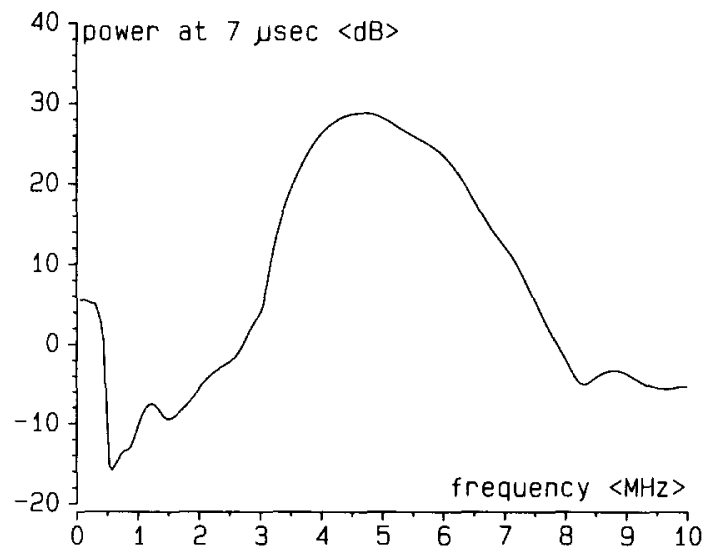

(b)

Fig. 1b. The power spectrum of the ultrasound reflection from the stainless steel reflector is the Fourier transform of the time domain signal depicted in Fig. 1a. This reference spectrum was used to correct the acquired myocardial ultrasound spectra. achieved by division of the measured power spectrum by the reference power spectrum for every frequency bin. Integrated backscatter was calculated by averaging the corrected power spectra over the useful frequency bandwidth from 3.2 to $7.2 \mathrm{MHz}$. Integrated backscatter levels were expressed in decibel units.

To measure the myocardial wall thickness, the acquired ultrasound signals of complete cardiac cycles were displayed in brightness mode on a video monitor using a $30 \mathrm{~dB}$ logarithmic signal compression. The endocardial wall was traced manually using the "mouse" of the computer system. Only the measurements of cardiac cycles having clearly visualized borders were selected for further analysis.

The time-dependent measurements of backscatter, percentage wall thickness and left ventricular pressure of the 10 consecutive cardiac cycles of every sequence were matched in time on the basis of the pressure curve. The time scales of the cardiac cycles were normalized to a linear scale expressed as a percentage of the cardiac cycle ranging from $0 \%$ (begin systole) to $100 \%$ (end diastole) with $2 \%$ increments. Mean values and standard deviations of the measurements were calculated for every $2 \%$ time increment of the cardiac cycle in every pig.

The measurements of integrated backscatter and wall thickness were related to their respective end-diastolic value taken during the baseline measurement interval in order to remove the intersubject differ- 
ences of backscatter levels from the data set (Rijsterborgh et al. 1990). In every pig the end-diastolic integrated backscatter level at baseline was defined as $0 \mathrm{~dB}$. Measurements of myocardial wall thickness were normalized with respect to the end-diastolic value measured during normal myocardial contractile performance and expressed as a percentage.

\section{Statistical analysis}

The relationship between relative backscatter and percentage wall thickening in normal myocardium was estimated by simple linear regression of the relative backscatter measurements and percentage wall thickening obtained during normal myocardial contractile performance for each pig.

The zero hypothesis was made that the relationship between backscatter and myocardial wall thickness as observed in normal myocardium is maintained during the state of acute ischemia. If a statistically significant additional contribution of the state of acute ischemia was found, the zero hypothesis was rejected.

On the basis of the estimated relationship, estimators of ischemic relative backscatter levels were calculated using the percentage wall thickening measured during acute ischemia in the same pig. The estimated relative integrated backscatter and the actual relative integrated backscatter measurements obtained in acutely ischemic myocardium were compared in the separate pigs using a paired $t$-test. A significance level of $p<0.01$ rejected the zero hypothesis. In every pig the mean values relative integrated backscatter measurements and their estimators were calculated, and a paired $t$-test was performed on the data as a group.

For visual inspection, combined scatterplots were made of the relative integrated backscatter versus percentage wall thickening of the measurements obtained during normal myocardial contractile performance and during acute ischemia.

\section{RESULTS}

A typical example of the measurements of relative integrated backscatter, percentage myocardial wall thickening and left ventricular pressure taken at baseline and during acute ischemia plotted versus percent cardiac cycle is in given in Fig. 2.

Combined scatterplots of the relative integrated backscatter measurements versus percentage wall thickening taken in the same pigs during baseline and during acute myocardial ischemia are depicted in Fig. 3.

The results of the simple linear regression between relative integrated backscatter and percentage myocardial wall thickening during normal contractile performance are given in Table 1 . The observed slopes in the individual pigs ranged from -0.163 $\mathrm{dB} / \%$ to $0.034 \mathrm{~dB} / \%$ (mean $=-0.036 \mathrm{~dB} / \%$, SD $=0.060 \mathrm{~dB} / \%$ ).

The mean values and standard deviations of the paired differences between the estimated relative integrated backscatter and the actually measured relative integrated backscatter including the results of the paired $t$-tests are given in Table 1 . In 15 out of 16 pigs a statistically significant contribution of ischemia was found and the zero hypothesis was rejected. The paired differences ranged from $-3.84 \mathrm{~dB}$ to $5.76 \mathrm{~dB}$ (group mean value $=0.31 \mathrm{~dB}, \mathrm{SD}=2.72 \mathrm{~dB}$ ). In eight pigs a positive contribution in $\mathrm{dB}$ was found whereas the other eight pigs showed a negative contribution. The additional contribution of ischemia to the backscatter measurements, although statistically significant, proved to be strongly dependent on the individual pig.

A paired $t$-test of the group mean differences resulted in a $p$-value of 0.65 (n.s.). Therefore, no predictable contribution of ischemia could be shown in the group of pigs. A scatterplot of this comparison is depicted in Fig. 4.

\section{DISCUSSION}

The cyclic variation of myocardial ultrasonic integrated backscatter has been shown to be a parameter of myocardial contractile performance (Wickline et al. 1985). An empirical relationship between integrated backscatter and myocardial wall thickness would explain this.

The purpose of this study was to investigate the relationship between integrated backscatter and wall thickening in an experimental animal model under normal and ischemic conditions. It should be realized that in acute ischemia the wall thickness of a pig's myocardium is decreased during most of the cardiac cycle as compared to its nonischemic end-diastolic value as can be appreciated from Fig. 2. These experiments were also designed to distinguish whether the increased backscatter levels during acute ischemia were related to a decreased wall thickness or the ischemia itself.

\section{The relationship between backscatter and wall thick-} ness

Myocardial ultrasonic backscatter is caused by the interaction between ultrasound and small structures within the myocardium. It is assumed that the size of these structures (scatterers) is small as compared to the wave length of the applied ultrasound 

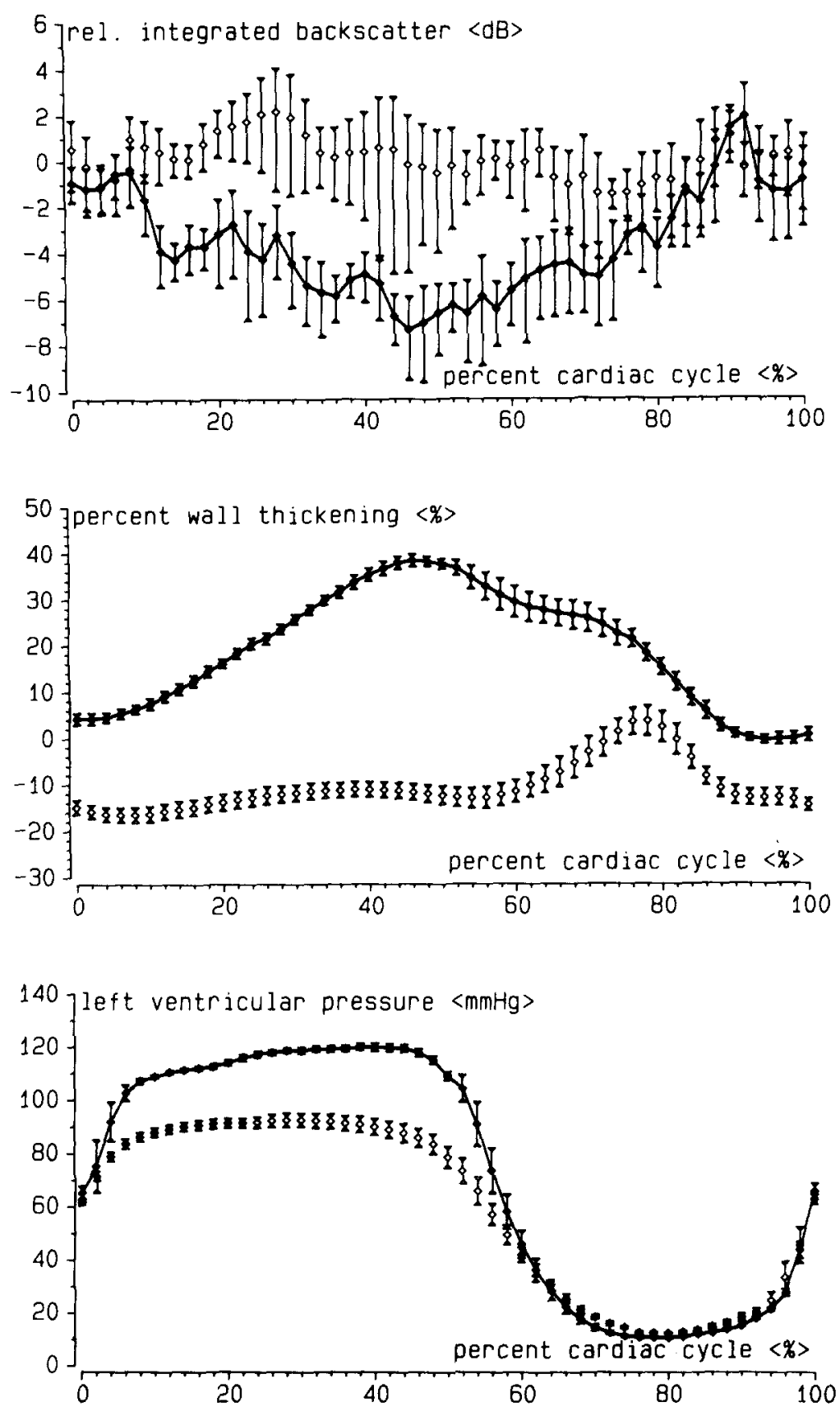

Fig. 2. The time-dependent measurements of relative integrated backscatter (top), percentage myocardial wall thickness (middle) and left ventricular pressure (bottom). Shown are the mean values (diamonds) and standard deviations (bars) of 10 cardiac cycles of pig no. 6 during baseline (connected with a solid line) and after 10 min subsequent acute ischemia.

frequency. Part of the acoustic energy is returned to the ultrasound transducer by these scatterers. According to the backscatter theory (Morse and Ingard 1968), the energy level of ultrasonic backscatter is determined by the density of the scatterers within the myocardium, the size of the scatterers and the frequency of the applied ultrasound. The change of myocardial integrated backscatter level at a given frequency during the cardiac cycle may therefore be explained by a change in scatter density or a change in scatter size or both. But the actual cause of the cyclic variation of myocardial integrated backscatter has not yet been assessed. Some information about this may be obtained by analyzing the corrected power 

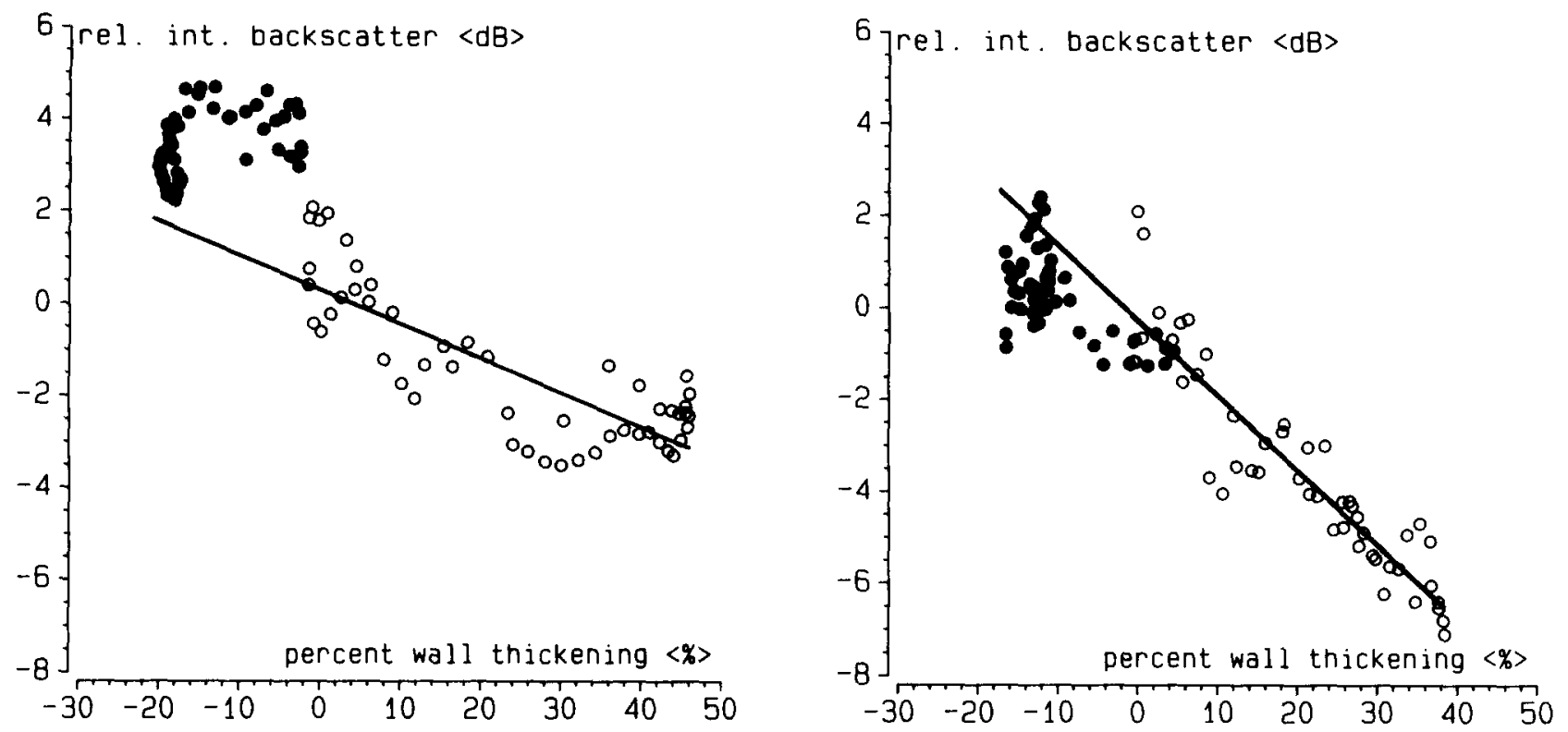

Fig. 3. Combined scatterplots of the relative integrated backscatter measurements versus percentage myocardial wall thickness taken in pig no. 1 (left-hand side) and in pig no. 6 (right-hand side). Shown are the mean values of 10 cardiac cycles. The line of regression was obtained by simple linear regression of the measurements taken at normal myocardial performance (open circles). The closed circles depict the measurements obtained during acute ischemia in the same pig.

Table 1. The slope and offset of the relationship between relative integrated backscatter (rel. IB) measurements and percentage myocardial wall thickness as determined by simple linear regression (columns 2 and 3). On the right-hand side the results are given of the paired $t$-tests between the estimated relative integrated backscatter based on this relationship and the actual measurements.

\begin{tabular}{|c|c|c|c|c|c|}
\hline \multirow[b]{2}{*}{$\begin{array}{l}\text { Pig } \\
\text { no. }\end{array}$} & \multicolumn{2}{|c|}{$\begin{array}{l}\text { Normal tissue: Rel. } \\
\text { IB versus \% wall } \\
\text { thickness }\end{array}$} & \multicolumn{3}{|c|}{$\begin{array}{l}\text { Acute ischemic tissue paired } \\
\text { differences: Actual rel. IB } \\
\text { minus estimated rel. IB }\end{array}$} \\
\hline & $\begin{array}{c}\text { Slope } \\
\text { (dB/\%) }\end{array}$ & $\begin{array}{l}\text { Offset } \\
\text { (dB) }\end{array}$ & $\begin{array}{c}\text { Mean } \\
\text { (dB) }\end{array}$ & $\begin{array}{l}\text { SD } \\
\text { (dB) }\end{array}$ & $t$-test \\
\hline 1 & -0.075 & 0.32 & 2.06 & 1.02 & $p<0.01$ \\
\hline 2 & -0.067 & -0.08 & -1.79 & 1.16 & $p<0.01$ \\
\hline 3 & 0.034 & 0.04 & 5.76 & 1.58 & $p<0.01$ \\
\hline 4 & 0.011 & 0.97 & 1.82 & 1.31 & $p<0.01$ \\
\hline 5 & -0.018 & -1.64 & 4.17 & 1.41 & $p<0.01$ \\
\hline 6 & -0.163 & -0.26 & -1.15 & 0.84 & $p<0.01$ \\
\hline 7 & 0.012 & -0.46 & 2.56 & 1.62 & $p<0.01$ \\
\hline 8 & 0.022 & -0.37 & 0.42 & 1.41 & $p=0.04$ \\
\hline 9 & -0.023 & 0.67 & -1.63 & 1.34 & $p<0.01$ \\
\hline 10 & 0.033 & -0.95 & -2.62 & 1.21 & $p<0.01$ \\
\hline 11 & 0.012 & 0.73 & -0.95 & 1.33 & $p<0.01$ \\
\hline 12 & -0.114 & 1.13 & -1.98 & 1.54 & $p<0.01$ \\
\hline 13 & -0.006 & -1.28 & 3.22 & 1.12 & $p<0.01$ \\
\hline 14 & -0.071 & 0.29 & 0.72 & 0.82 & $p<0.01$ \\
\hline 15 & -0.122 & 0.53 & -3.84 & 0.97 & $p<0.01$ \\
\hline 16 & -0.041 & 0.02 & -1.82 & 1.82 & $p<0.01$ \\
\hline mean & -0.036 & -0.02 & 0.31 & & \\
\hline SD & 0.060 & 0.79 & 2.72 & & \\
\hline
\end{tabular}

spectrum (backscatter transfer function). Since integrated backscatter is the frequency average of the backscatter transfer function, the information on whether the cyclic variation is caused by change in scatter density or by a change in scatter size is lost. This is currently under investigation.

The empirical relationship between myocardial integrated backscatter and myocardial wall thickness has been studied in animal experiments before (Wickline et al. 1986; Rijsterborgh et al. 1990) but not to a large extent. The result of the present study showed an average slope of $-0.036 \mathrm{~dB} / \%$. This would result in a mean cyclic variation of $1.8 \mathrm{~dB}$ for a $50 \%$ change in wall thickness. An interesting observation of this study is that the ranges of the estimated slopes proved to be quite large $(-0.163 \mathrm{~dB} / \%$ to 0.034 $\mathrm{dB} / \%$ ). This indicates that there are consistent individual differences between the pigs, notwithstanding the controlled conditions under which this in vivo study was performed. Since the ultrasound transducer was sutured directly upon the pig's myocardium, the integrated backscatter measurements were marginally influenced by a change of the angle of insonification during the cardiac cycle. Secondly, these measurements are not corrupted by an unknown frequency-dependent attenuation of the ultrasound path in between the transducer and the re- 


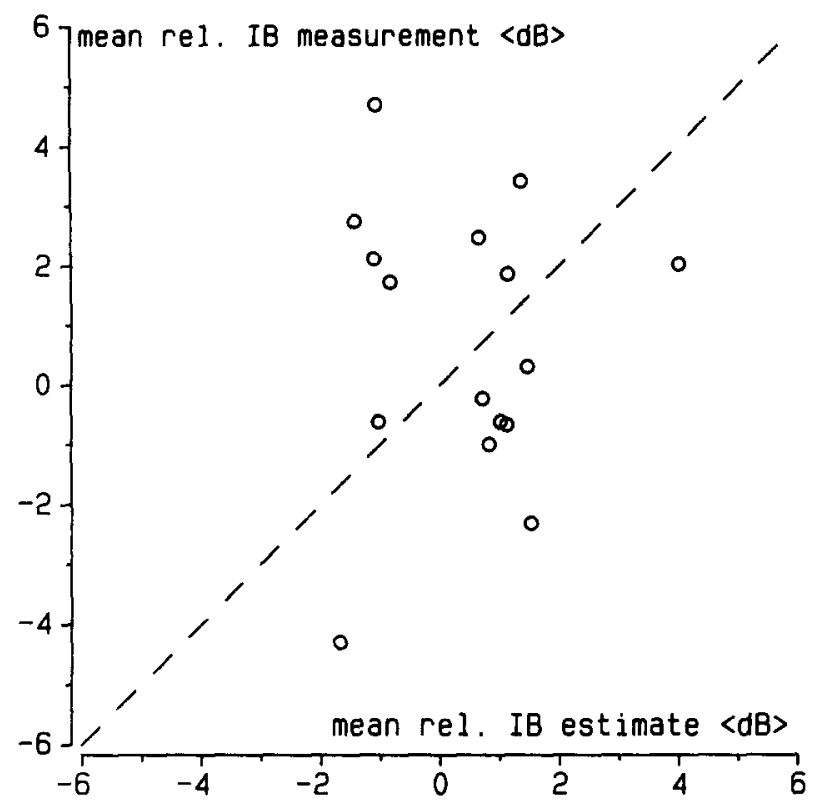

Fig. 4. Scatterplot of the mean values of the estimated relative integrated backscatter (rel. IB) measurements versus the actually measured relative integrated backscatter obtained in 16 pigs. A measurement above the line of identity (dashed line) signifies a positive contribution of the state of acute ischemia to the relative integrated backscatter measurement.

gion of interest opposite to closed-chested in vivo experiments.

\section{The additional contribution of ischemia}

Fifteen out of 16 pigs showed statistically significant contributions to the integrated backscatter levels by ischemia. However, the contribution does not appear to be consistent in the individual pig: $50 \%$ of the pigs examined showed a positive contribution, the other $50 \%$ a negative contribution. Therefore, other parameters than myocardial wall thickness and ischemia must be involved. However, since no overall contribution of ischemia could be proven in the analysis of the data as a group, the unknown factor also appeared to cancel out. The hypothesis that the relationship between integrated backscatter and wall thickness is maintained during acute ischemia of the myocardium was rejected in all but one of the individual pigs but not in the group as a whole.

\section{The variability of myocardial integrated backscatter measurements}

In a previous study (Rijsterborgh et al. 1990), we reported a large intersubject variation of absolute integrated backscatter levels in the myocardium of different pigs. In the current study, we observed a large intersubject variation of the relationship between integrated backscatter and myocardial wall thickness during normal contractile performance. Three different explanations may be given here. By applying the backscatter theory to these observations, one has to conclude that there are individual differences in the structure of a pig's myocardium.

A second explanation may be individual differences in layout of the coronary arteries of a pig's heart. In the experiments the position of the ultrasound transducer was referenced to the actual layout of the LADCA. Therefore, small differences in position of the transducer with regard to the outer geometry of the heart may be introduced.

A third explanation may be that in practice the ultrasound signals which are returned by the pig's myocardium consist of a combination of backscatter signals and weak specular reflections. In the time domain, one cannot distinguish genuine backscatter signals originating from scatterers smaller than the wave length in size, and weak specular reflections caused by structures larger than the wave length. Analysis of the backscatter transfer function of myocardial tissue may solve this problem. This should be a subject for further investigation.

\section{CONCLUSION}

The increased myocardial integrated backscatter level during acute ischemia is more related to the wall thickness than to the state of acute ischemia itself. On the average, there is no consistent additional contribution to the level of integrated backscatter measurements if the dependency of wall thickness is removed from the data.

In addition, it may be concluded that integrated backscatter measurements in pigs are subjected to a large intersubject variation. In normal myocardium, the slope relationship between myocardial integrated backscatter level and wall thickness shows a large variation.

\section{REFERENCES}

Bloomfield, P. Fourier analysis of time series: An introduction. New York: John Wiley; 1976:80-85.

Glueck, R. M.; Mottley, J. G.; Miller, J. G.; Sobel, B. E.; Pérez, J. E. Effects of coronary artery occlusion and reperfusion on cardiac cycle-dependent variation of myocardial ultrasonic backscatter. Circ Res 56:683-689; 1985.

Lancée, C. T.; Mastik, F.; Rijsterborgh, H.; Bom, N. Myocardial backscatter analysis in animal experiments. Ultrasonics 26:155-163; 1988

Miller, J. G.; Pérez, J. E.; Mottley, J. G.; Madaras, E. I.; Johnston, P. H.; Blodgett, E. D.; Thomas, L. J.; Sobel, B. E. Myocardial tissue characterization: An approach based on quantitative backscatter and attenuation. Proc. IEEE Ultrasonics Symp. 83 CH1947-1:782-793; 1983. 
Morse, P. M.; Ingard, K. U. Theoretical acoustics. New York: McGraw-Hill Company; 1968:400-441.

Mottley, J. G.; Glueck, R. M.; Pérez, J. E.; Sobel, B. E.; Miller, J. G. Regional differences that parallel regional differences in contractile performance. J. Acoust. Soc. Am. 76:1617-1623; 1984.

O'Donnell, M.; Bauwens, D.; Mimbs, J. W.; Miller, J. G. Broadband integrated backscatter: An approach to spatially localized tissue characterization in vivo. Proc. IEEE Ultrasonics Symp. 79 CH1482-9;175-178; 1979.

Office of Science and Health Reports. Guide for the care and use of laboratory animals. DHEW Publication No. (NIH)80-23, DRR/NIH Bethesda, Maryland; 1980.

Rijsterborgh, H.; Mastik, F.; Lancée, C. T.; van der Steen, A. F. W.; Sassen, L. M. A.; Verdouw, P. D.; Roelandt, J.; Bom, N. Ultra- sonic myocardial integrated backscatter and myocardial wall thickness in animal experiments. Ultrasound in Med. \& Biol. 16:29-36; 1990.

Sassen, L. M. A.; den Boer, M. O.; Rensen, R. J.; Saxena, P. R.; Verdouw, P. D. B-isoprolol improves perfusion of ischaemic myocardium in anaesthetized pigs. Br. J. Pharmacol. 95:361$370 ; 1988$.

Wickline, S. A.; Thomas III, L. J.; Miller, J. G.; Sobel, B. E.; Pérez, J. E. The dependence of myocardial ultrasonic integrated backscatter on contractile performance. Circulation 72:183-192; 1985.

Wickline, S. A.; Thomas III, L. J.; Miller, J. G.; Sobel, B. E.; Pérez, $J$. E. Sensitive detection of the effects of reperfusion on myocardium by ultrasonic tissue characterization with integrated backscatter. Circulation 74:389-400; 1986. 